\title{
Uma abordagem sobre a integração da Computação de Borda Móvel e a Rede 5G para Internet das Coisas na Agricultura 4.0
}

\author{
Kaíque M. R. Cunha ${ }^{1}$, Rúben F. Xavier ${ }^{1}$, Waldir Moreira ${ }^{3}$, \\ Leandro A. Freitas ${ }^{2}$ e Antonio Oliveira-Jr ${ }^{1,3}$ \\ ${ }^{1}$ Instituto de Informática (INF) - Universidade Federal do Goiás (UFG) \\ Alameda Palmeiras, Quadra D, Câmpus Samambaia \\ 74.69-900 - Goiânia - GO - Brazil \\ ${ }^{2}$ Instituto Federal de Goiás \\ Núcleo de Estudos aplicados a Redes de computadores e \\ Sistemas distribuídos (NumbERS) \\ Avenida Universitária, $\mathrm{s} / \mathrm{n}^{\circ}$, Vale das Goiabeiras \\ 75.400-000 - Goiânia - GO - Brazil \\ ${ }^{3}$ Fraunhofer Portugal AICOS \\ Porto, Portugal \\ \{kaiquematheus, rubenxavier\}einf.ufg.br \\ leandro.freitas@ifg.edu.br, antoniojr@ufg.br, waldir.junior@fraunhofer.pt
}

\begin{abstract}
Agriculture 4.0 has been gaining strength in the Brazilian market using technologies such as the Internet of Things, mobile edge location, $5 G$ networks, boosting agricultural development and the country's economy. With the use of different technologies and interconnected devices, a large amount of data and stringent requirements grows, meeting various demands such as high speed, security, reliable communication, among others. This article proposes a MultiAccess Edge Computing (MEC) integration with the $5 G$ Network and proposes a Radio Network Information Service (RNIS), which will be implemented in the $M E C$ architecture as a way to provide requirements for Agriculture 4.0 applications.
\end{abstract}

Resumo. A agricultura 4.0 vem ganhando força no mercado brasileiro utilizando de tecnologias como Internet das Coisas, computação de borda móvel, Redes 5G, para impulsionar o desenvolvimento agropecuário e a economia do país. Com a utilização de diversas tecnologias e dispositivos interligados, cresce a grande quantidade de dados e requisitos rigorosos necessários para atender diversas demandas tais como alta velocidade, segurança, comunicação confiável, entre outros. Este artigo propõe a integração do Multi-Access Edge Computing (MEC) com a Rede $5 G$ e propõe um serviço de Radio Network Information Service (RNIS), que será implantado na arquitetura MEC como forma de prover requisitos para aplicações da Agricultura 4.0.

\section{Introdução}

O agronegócio vem se tornando a décadas uma das principais fontes econômicas do Brasil [Martins and Barbosa 2019]. As condições climáticas, extensão territorial, alto potencial 
produtivo e os esforços de instituições públicas e privadas direcionadas ao desenvolvimento tecnológico e científico tornam o Brasil um dos maiores produtores e exportadores agrícolas do mundo [Martins and Barbosa 2019]. Contudo, este setor se torna cada vez mais competitivo com novos desafios e conceitos capazes de revolucionar o agronegócio do Brasil e do mundo.

A agricultura 4.0 (agro 4.0) carrega conceitos e métodos da Indústria 4.0 [Silva and Cavichioli 2020, Massruhá and Leite 2017]. Neste contexto, passa-se a utilizar diversos dispositivos com o objetivo de melhorar o cenário da agricultura, impulsionando o uso de sensores e mecanismos estratégicos na agricultura, automação e robótica agrícola [Silva and Cavichioli 2020, Massruhá and Leite 2017].

A Internet das Coisas (Internet of Things - IoT) une o mundo real e digital, permitindo que indivíduos possam interagir constantemente com outras pessoas e objetos [Silva and Cavichioli 2020, Martins and Barbosa 2019]. Deste modo, há uma mudança na forma como interagimos com o mundo físico, viabilizando medidas computacionais, permitindo novos negócios, promovendo segurança, gerenciamento de infraestrutura pública, agrícola e outros [Massruhá and Leite 2017]. Associada a tecnologias como as redes de quinta geração (5G), computação em nuvem e em borda, a IoT reflete o futuro em que diversas soluções impulsionam o avanço agrícola e industrial [Massruhá and Leite 2017].

Portanto, a agro 4.0 usará tecnologias avançadas da computação como conectividade entre dispositivos móveis, métodos computacionais de alto desempenho, computação em nuvem, IoT, 5G e sensoriamento agrícola, para potencializar o setor [Silva and Cavichioli 2020, Massruhá and Leite 2017]. A melhora na tomada de decisão, manejo, estratégias agrícolas e segurança, automação de serviços, aumento na produtividade e eficiência no uso de insumos, redução dos impactos ambientais são algumas das possibilidades do agro 4.0 [Silva and Cavichioli 2020, Massruhá and Leite 2017]. As tecnologias estão ligadas a todos os setores da cadeia produtiva, de modo a impulsionar o agronegócio. Neste contexto, as operações e tomadas de decisão passam a ser feitas a partir de informações coletadas em tempo real, referente ao clima, terra, lavoura, gado, produção [Massruhá et al. 2020, Massruhá and Leite 2017].

A implantação de serviços avançados de IoT requer requisitos de comunicação rígidos que serão alcançados pelo $5 \mathrm{G} / 6 \mathrm{G}$, colocando a computação de borda no universo das soluções para a agricultura e indústria [Wang et al. 2018, Sabella et al. 2016]. A computação de borda, permite o processamento de dados na borda da rede, mais próximo do usuário final [Hu et al. 2015].

Este trabalho tem como objetivo abordar os principais conceitos relevantes para a agricultura 4.0, propondo a integração do Multi-Access Edge Computing (MEC) e a Rede 5G para IoT. Propomos um serviço de Radio Network Information Service (RNIS) que será implantado na arquitetura MEC para otimizar aplicações de borda no cenário da agro 4.0, por meio de informações de Radio Access Network (RAN) obtidas através do serviço RNIS. Com essas informações, objetiva-se contribuir com o desempenho e performance das aplicações agrícolas. A partir disso, realizou-se o deployment completo do framework MEC EALTEdge e também do núcleo 5G my5G-core, que faz parte da iniciativa my5G, ambos em ambiente virtualizado. Criou-se um setup de testes a fim de 
validar a comunicação do framework EALTEdge com a Internet através do my5G-core. As principais contribuições deste artigo são:

- Deployment do framework EALTEdge e my5G-core;

- Integração MEC - 5G;

- Proposta de serviço RNIS implantado na arquitetura MEC como forma de prover requisitos para aplicações da Agro 4.0;

O artigo está organizado da seguinte forma. A Seção 2 contextualiza a Agricultura 4.0 com seus casos de uso. Já a Seção 3 apresenta os principais conceitos deste trabalho. A Seção 4 apresenta a proposta de desenvolvimento RNIS para Agricultura 4.0. A Seção 5 apresenta os resultados deste trabalho. Por fim, a Seção 6 apresenta a conclusão e os próximos passos deste trabalho.

\section{Agricultura 4.0}

De acordo com Centro de Estudos Avançados em Economia Aplicada (CEPEA) e a Confederação da Agricultura e Pecuária do Brasil (CNA), o agronegócio é responsável por cerca de 26\% do PIB nacional em 2020 [Cepea 2020]. O Brasil é um dos maiores exportadores de alimentos do mundo [Ferreira et al. 2019]. Dos seus 851,5 milhões de hectares de área total, 69 milhões de hectares são utilizados para agricultura, destacandose no fornecimento de soja, carnes, café, dentre outros [Ferreira et al. 2019]. Mesmo com destaque, o Brasil tem potencial de crescimento para ampliar a eficiência e produtividade no meio agrícola. Neste cenário, tecnologias ligadas à IoT se revelam uma importante alternativa para o aumento da eficiência e produtividade da agropecuária brasileira [Ferreira et al. 2019]. Viabilizando diversas medidas computacionais promovendo segurança, permitindo novos negócios, gerenciamento de infraestrutura pública e agrícola [Massruhá and Leite 2017].

Estudos revelam que o ganho econômico no ambiente rural global impulsionado pela IoT pode chegar a US\$ 61 a US\$ 362 bilhões em 2025 [Ferreira et al. 2019]. No Brasil, com a adoção destas tecnologias, o ganho econômico no mesmo ambiente rural é da ordem de US\$ 5,5 a US\$ 21,1 bilhões em 2025 [Ferreira et al. 2019].

Massruhá e Leite (2017) ressalta que a Empresa Brasileira de Pesquisa Agropecuária (EMBRAPA) tem como desafio desenvolver pesquisas para integrar diversas tecnologias, produzindo conhecimento para que o Brasil continue sendo um protagonista na produção e exportação agropecuária, visando aumentar a produtividade alimentícia. Cada vez mais as tecnologias da informação e da comunicação (TIC) impulsionam a inovação dentro e fora da cadeia produtiva, utilizando aplicações para auxiliar na agricultura de precisão e diversos cenários.

As melhorias e inovações de tecnologias para transmissão, análise e processamento de dados, assim como nas telecomunicações, são de suma importância para que o produtor rural possa se desenvolver, adquirir competitividade e sustentabilidade [Silva and Cavichioli 2020]. Além do surgimento de diversas ferramentas e práticas, a agro 4.0 proporcionará aumento da produtividade [Bonneau et al. 2017]. A chave dessa transformação está na capacidade de coletar mais informações e medições sobre a produção, clima, umidade e qualidade do solo, são exemplos de dados que serão obtidos a partir de sensores e equipamentos espalhados na área agrícola [Bonneau et al. 2017]. 
A fim de obter tecnologias que possibilitem a melhoria do setor agrícola, são enfrentados desafios tais como delay, baixa qualidade de transmissão de sinal e baixa capacidade de processamento. Isto exige que as redes atendam as aplicações de forma flexíveis para suportar os diferentes requisitos [Ferreira et al. 2019].

\subsection{Casos de Uso}

No cenário da agro 4.0, são abordados diversos conceitos como por exemplo a agricultura inteligente (smart farming) que utiliza-se de informações para tomada de decisões, com a finalidade executar tarefas e controlar processos automatizados em diversas atividades agrícolas [Ferreira et al. 2019]. O gerenciamento inteligente de informações envolve o constante monitoramento dos eventos ligados a um sistema inteligente, que possibilita a tomada de decisões e organização dinâmica de atividades agrícolas em situações como: mudança climática ou detecção de pragas e doenças [Ferreira et al. 2019]. Ferreira et al. (2019) destaca algumas aplicações IoT que podem potencializar a produtividade e eficiência da agropecuária, alguns exemplos são:

- Sistemas de identificação, controle de pragas e doenças: A captura de imagens processadas por Inteligência Artificial (IA) pode ser uma das soluções, identificando por exemplo o indício de doenças, presença e a quantidade de agentes indesejados. Possibilitando assim o aumento na qualidade e redução da perda de produtos, propagação de doenças e pragas, entre outros;

- Sistemas de monitoramento meteorológico: A IoT possibilita que sensores climáticos e meteorológicos sejam instalados em diferentes locais, proporcionando o monitoramento da temperatura, mensuração da velocidade e identificar a direção do vento, detecção de chuvas, dentre outros parâmetros em tempo real;

- Prevenção de desastres: Desastres como deslizamento de encostas e barrancos, incêndio em áreas críticas podem causar grandes impactos ambientais, econômicos e sociais. A velocidade na comunicação e monitoramento em tempo real são alguns dos requisitos primordiais para a prevenção de desastres;

- Aplicação de robôs na agricultura: É uma alternativa para a falta de mão de obra e otimização da produtividade na agricultura. Os robôs na agricultura utilizam de algumas tecnologias como processamento de imagens, aprendizado de máquina e inteligência artificial, para que percebam o ambiente ao seu redor e se adaptem a ele. Esta utilização ainda se encontra em estágio inicial, tendo como principais casos de uso: i) Colheita de frutas; ii) Pulverização e remoção de ervas daninhas; iii) Drones e imagens aéreas;

A quantidade de novas informações geradas pelas redes IoT, bem como a utilização de técnicas como a IA e Big Data Analytics, permitirão um universo de novas aplicações [Ferreira et al. 2019]. As aplicações IoT exigem diversos requisitos sobre as redes como altas taxas de dados e baixa latência, transmissão de vídeos em alta resolução em tempo real, análise e respostas ultra confiáveis em tempo real. Com diversas demandas e necessidades exigentes, as redes devem ser flexíveis o suficiente para suportar diversos requisitos [Ferreira et al. 2019]. Na Figura 1, baseada em Fuentes et al. (2021), é possível visualizar o cenário do agro 4.0, o qual possui diversos sensores espalhados no ambiente como por exemplo sensores para o monitoramento remoto, controle de máquinas pesadas, localização e análise de rebanho e plantio. Os sensores espalhados necessitam de requisitos de comunicação rígidos e informações como o contexto do ambiente. Assim, tais 


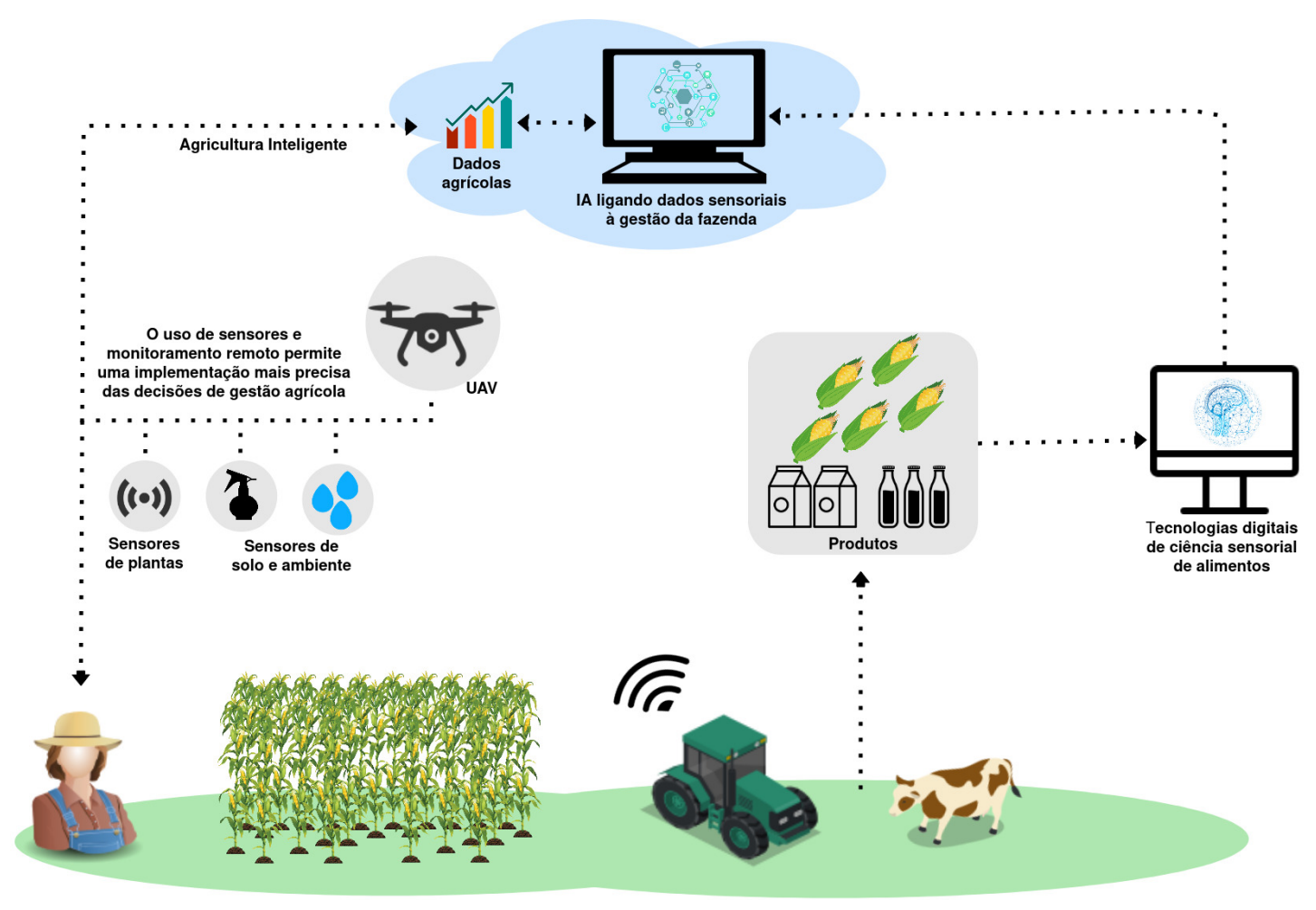

Figure 1. Cenário da Agro 4.0 Baseado em [Fuentes et al. 2021]

informações são reunidas na borda da rede e tratadas para cada aplicação, auxiliando na tomada de decisão de qualidade dos serviços.

\section{Conceitos}

\subsection{Redes 5G}

A arquitetura do sistema 5G (5G System - 5GS) especificada pelo 3rd Generation Partnership Project (3GPP) e descrita em Rommer et al. (2019), foi projetada para atender a um amplo conjunto de casos de uso que vão desde uma grande quantidade de dispositivos IoT, até o outro extremo de elevada taxa de bits e serviços de missão crítica de alta confiabilidade. Esta arquitetura pode ser vista na Figura 2. O suporte a todos os casos de uso com a mesma arquitetura comum exigiu mudanças significativas no design tanto para a RAN quanto para o núcleo da rede [ITU 2021].

Uma das principais mudanças em relação as gerações anteriores é a nova arquitetura construída sob um conceito chamado Service-Based Architecture (SBA), que é baseada em serviços e microsserviços [3rd Generation Partnership (3GPP) 2018]. A principal mudança presente nesta nova arquitetura é a separação entre o plano de dados (data plane - DP) e o plano de controle (control plane - CP), além disto, também traz como característica a virtualização de funções (network function virtualization - NFV) e fatiamento de rede (network slicing - NS) [Silva et al. 2021]. O SBA oferece vantagens a arquitetura 5G, como maior eficiência na manutenção e desenvolvimento, associação de microsserviços a recursos exclusivos e ciclos de vida independentes, e melhor escalabilidade com instanciação sob demanda de serviços [Rommer et al. 2019]. 


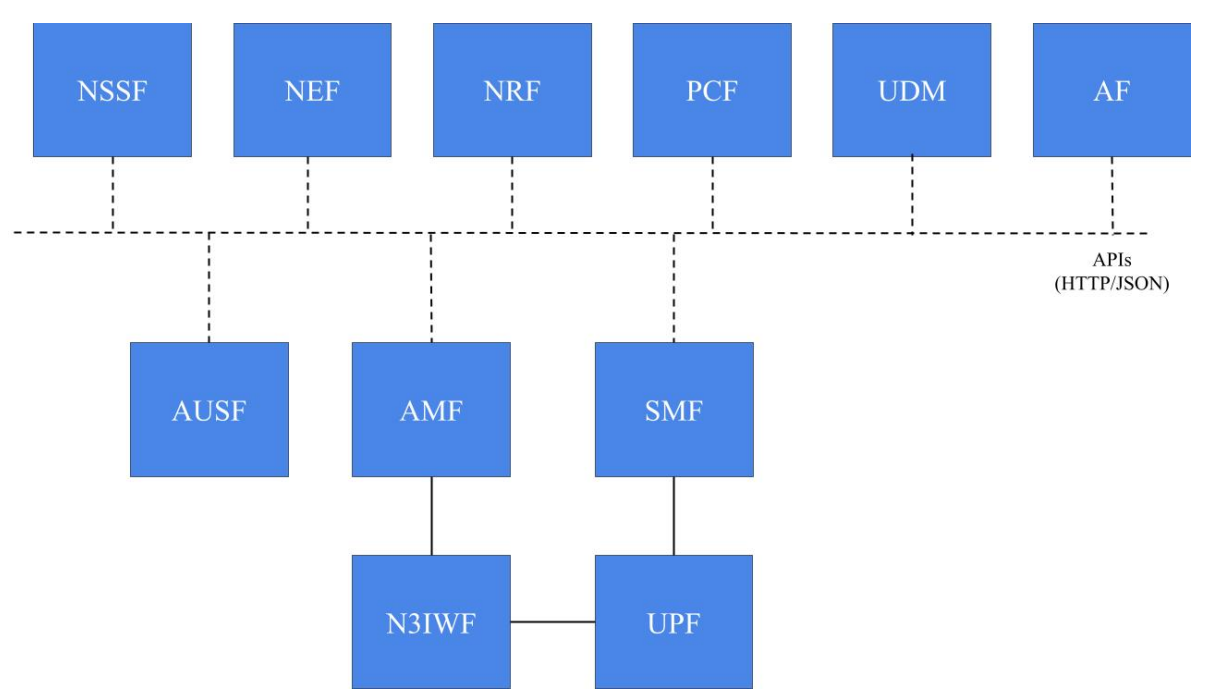

Figure 2. Arquitetura do 5GC. Baseada em [Silva et al. 2021]

A 3GPP continua atualizando seus documentos técnicos, sempre com foco na expansão do sistema 5G. Isto é feito a partir da publicação de novas releases. Exemplo disto, a release 16 definiu serviços prioritários de multimídia, serviços da camada de aplicativos em Vehicle-to-everything (V2X), acesso via satélite, acesso a rede local, convergência sem fio e cabeada, posicionamento e localização de terminais de comunicações em domínios verticais e automação de rede [SILVA et al. 2020]. Em todos estes contextos, 5G deve oferecer desempenho operacional consideravelmente maior como taxa de dados mais altas, baixa latência, melhor experiência de usuário, atendendo a imensa implantação de novos dispositivos, oferecer níveis de consumo de energia aceitáveis, custos de equipamento e implantação de rede e custo de operação [ETSI 020a].

O 5GC é parte do 5GS juntamente com uma nova interface de radio (New Radio NR). Com base nos conceitos de computação em nuvem, o 5GS tem o seu núcleo orientado a serviços com a arquitetura SBA, além de suporte nativo a fatiamento de recursos de rede, virtualização e computação móvel de borda [Foukas et al. 2017].

\subsection{Multi-Access Edge Computing (MEC)}

Introduzido pelo European Telecommunications Standards Institute (ETSI), o MEC veio como uma forma de estender a inteligência para o limite da rede com maior capacidade de processamento e armazenamento [Porambage et al. 2018]. O órgão de pesquisa 5G Infrastructure Public Private Partnership (5G PPP) reconhece o MEC, NFV e SoftwareDefined Networking (SDN) como uma das principais tecnologias emergentes para redes 5G [Association et al. 2015].

As redes $5 \mathrm{G}$ impulsionaram abordagens programáveis para redes de software usando a tecnologia de virtualização de TI amplamente na infraestrutura, funções e aplicativos de telecomunicações [Hu et al. 2015]. Estendendo recursos computacionais em nuvem até o limite das redes é possível minimizar o congestionamento da rede melhorando a otimização de recursos, desempenho e a experiência do usuário [Porambage et al. 2018]. Com a utilização da RAN, o MEC proporciona melhoria na latência e na utilização da largura de banda, tornando os serviços mais acessíveis a desenvolvedores e provedores de conteúdo [Porambage et al. 2018]. 
O MEC é uma tecnologia chave que permitirá a evolução para o 5G, avançando na transformação de rede de banda larga móvel em um mundo programável e contribuindo para os requisitos exigentes do IoT e 5G como latência, escalabilidade e outras [Hu et al. 2015]. "O princípio básico do MEC é estender os recursos de computação em nuvem até o limite das redes celulares" [Hu et al. 2015]. O MEC proporciona um ambiente de serviços de TI e recursos de computação em nuvem na borda da rede móvel dentro da RAN, com objetivo de oferecer uma experiência de usuário reduzindo a latência, garantindo operações de rede e entrega de serviços com alta eficiência [Porambage et al. 2018].

A IoT foi identificada como um dos principais casos de uso do MEC pelo ETSI [Hu et al. 2015]. O MEC permite a implantação de serviços versáteis e contínuos em aplicativos IoT, capacitando os com recursos computacionais e da mesma forma a IoT expande os serviços MEC para diversos tipos de objetos inteligentes, assim o MEC e IoT trazem vantagens um ao outro [Porambage et al. 2018].

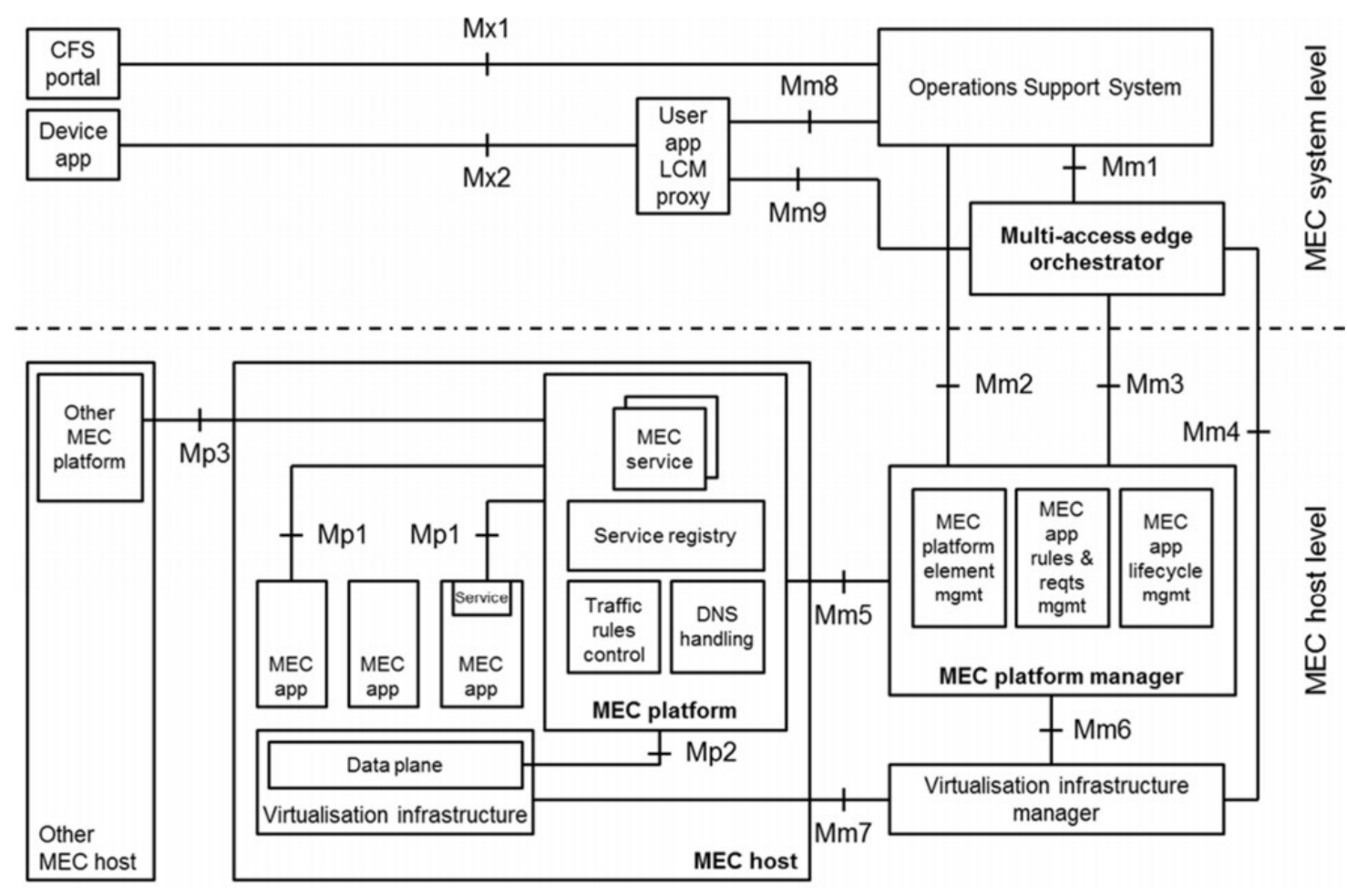

Figure 3. Arquitetura de referência MEC. Retirada de [ETSI 020b]

A arquitetura de referência MEC é a base para o desenvolvimento de qualquer framework MEC. Esta está definida em [ETSI 020b] e pode ser vista na Figura 3. Nela observa-se todos os componentes MEC, dentre eles, quatro principais:

- MEC platform (MEP): Atua como uma interface entre a rede móvel e os aplicativos MEC, ou seja, é a coleção de funcionalidades essenciais necessárias para executar aplicativos MEC em uma determinada infraestrutura de virtualização, permitindo que forneçam e consumam serviços MEC [ETSI 020b];

- MEC host: É uma entidade que contém um MEP e uma infraestrutura de 
virtualização que fornece computação, armazenamento e recursos de rede, para fins de execução de aplicações MEC [ETSI 020b];

- MEC applications (MEC APPs): Instâncias executadas no topo da Network Functions Virtualization Infrastructure (NFVI) do MEC host, com base na configuração ou até solicitações realizadas pelo MEC;

- Multi-Access Edge Orchestrator (MEO): É responsável pelo gerenciamento do ciclo de vida dos aplicativos MEC, atuando como interface entre o MEC host e o Operations/Business Support System (OSS/BSS) da operadora [ETSI 020b], [Ksentini and Frangoudis 2020].

\section{Serviço RNIS}

Presume-se que as redes 5G enfrentarão um aumento no volume de tráfego e em suas demandas computacionais. Isso se dá devido a popularização de aplicativos, serviços e atividades computacionais que exigem alto poder computacional [Pham et al. 2020]. A maioria dos dispositivos computacionais possuem capacidade de armazenamento e processamento limitado, surgindo problemas recorrentes [Pham et al. 2020]. Um exemplo disto é como executar tarefas que exigem alto poder computacional em dispositivos com recursos escassos [Pham et al. 2020].

Neste contexto, o MEC é visto como uma tecnologia fundamental nas redes 5G, permitindo a otimização de recursos móveis hospedando serviços de computação intensiva e fornecendo recursos computacionais em cloud. Isto aproxima dispositivos computacionais, oferece serviços sensíveis ao contexto com informações proporcionadas pela RAN [Pham et al. 2020].

Anteriormente, as informações em nível de RAN só estavam disponíveis para operadoras de rede por meio de interfaces de monitoramento dos equipamentos móveis. Atualmente, o acesso a essas informações está disponível ao MEC através do serviço de RNIS [Arora et al. 2019]. O RNIS é responsável por interagir com a RAN, coletando informações de rádio referentes aos equipamentos de usuários (user equipments - UE) [Arora et al. 2019]. Isto permite que informações como latência, localização, força do sinal, sejam utilizadas de maneiras diferentes, de acordo com a necessidade das aplicações, permitindo o ajuste dinâmico de seu comportamento, melhorando e otimizando o seu desempenho perante as condições da RAN [Arora et al. 2019] [ETSI 2019].

Este aspecto proporciona uma abertura para desenvolvimento de novos serviços ligados à RAN. Executando na borda, soluções baseadas em qualidade de experiência (quality of experience - QoE), gerenciamento de recursos da rede, auxílio na tomada de decisão e otimização de serviços serão contemplados [Arora et al. 2019]. O serviço RNIS possibilita que diversas aplicações acessem informações de acordo com seu contexto. Deste modo, poderão aprimorar a oferta de seus serviços e desempenho [Arora et al. 2019].

Os dados de uma RAN são gerados em grande escala e têm a necessidade de serem tratados. Executar estas operações em dispositivos que recursos como armazenamento, processamento são limitados é um desafio [Arora et al. 2019]. Os desafios de escalabilidade surgem conforme o número de terminais móveis geram dados e o número de aplicativos MEC-hosted, que consomem o RNIS, aumenta [Arora et al. 2019]. 
Informações de nível RAN fornecidos pelo RNIS como congestionamento da rede, latência, força do sinal, atualização de localização dos dispositivos na rede, indicação da qualidade do canal, podem auxiliar no monitoramento de gado, controle de pragas e doenças, identificação de desastres e utilização de robôs no campo, além de beneficiar a QoE dos UEs. Assim, os MEC APPs autorizados a utilizarem essas informações podem otimizar o tráfego do usuário, em casos de congestionamento da rede ou alta latência o usuário deve ser capaz de mudar para outra rede sem problemas, sem que afete a QoE. Por exemplo, as informações de taxa de transferência podem ajudar servidores de vídeo a tomar decisões de controle de congestionamento TCP. O mesmo pode ser feito na tomada de decisão em aplicações com base na prevenção de desastres. Essas informações podem ser usadas para garantir que aplicativos correspondam à capacidade estimada no downlink da rede de rádio [Tan et al. 2018].

Com a revisão bibliográfica feita até o momento, embora alguns trabalhos se concentram nas aplicações do RNIS, não foram encontrados trabalhos voltados para a abordagem de implementação do RNIS em um framework MEC integrado a Rede 5G que utilize o RNIS para contribuir com o desempenho e performance de aplicações voltadas para o agronegócio. Um dos trabalhos mais próximos é o de Arora et al. (2019) no qual são implementadas soluções alternativas para a distribuição de mensagens no modelo publicar-assinar (RabbitMQ e Apache Kafka). Avaliando e comparando o desempenho duas soluções para a implementação do RNIS no MEC. Portanto surge a proposta de um serviço RNIS implantado na arquitetura MEC integrada a Rede 5G, como forma de prover requisitos (quaisquer informações pertinentes) para aplicações da agro 4.0.

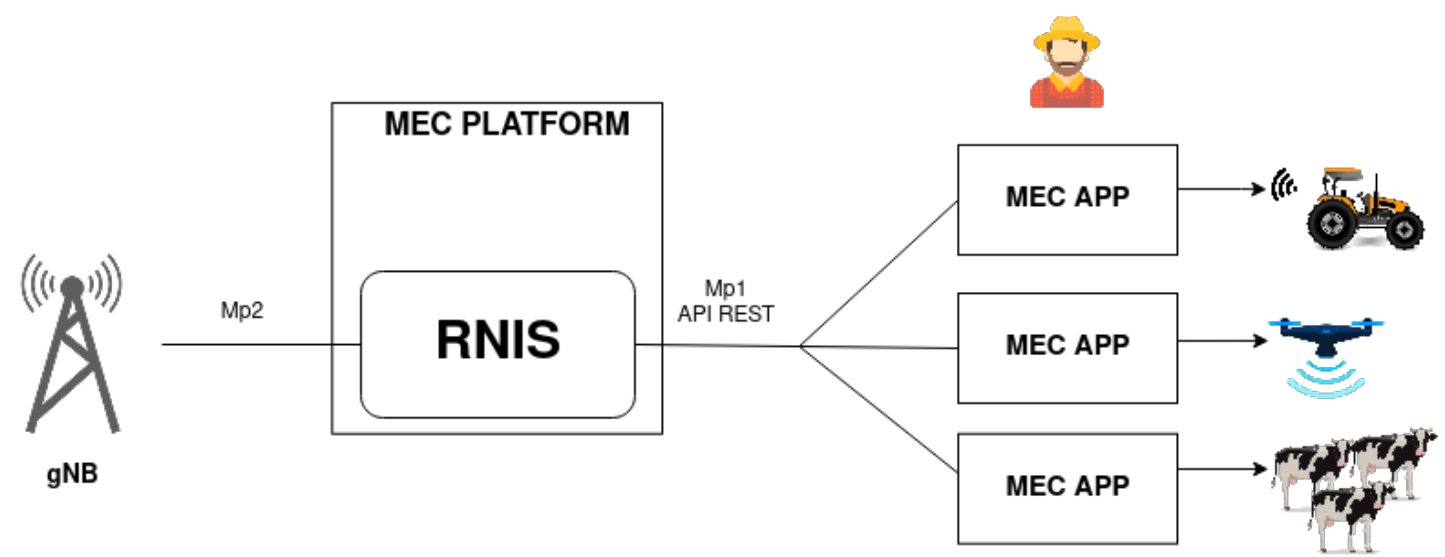

Figure 4. RNIS Baseado em [Arora et al. 2019, ETSI 020b]

Como ilustrado na Figura 4 baseada em Arora et al. (2019) e ETSI GS MEC 012 V2.1.1 [ETSI 020b], propomos a implementação de um serviço RNIS implantado ao MEC platform, utilizando a arquitetura de referência MEC apresentada na Figura 3 e também propomos a integração da arquitetura MEC a Rede 5G. Deste modo o serviço RNIS disponibiliza informações com granularidades ajustáveis aos MEC APPs com casos de uso na agro 4.0. Afim de contribuir com o desempenho e performance das aplicações agrícolas. Para a implantação do serviço RNIS, deverá ser implementada a interface $M p 2$ que permite a integração com o Next Generation NodeB (gNodeB, gNB), necessário para gerenciamento de tráfego, direcionamento para MEC APPs e para reunir 
informações de nível RAN sobre o ambiente e contexto dos UEs agrícolas, que serão expostos [Arora et al. 2019]. Para a comunicação e disponibilização de informações personalizadas, o RNIS se comunica através da interface $M p 1$ que permite ao MEC APPs se inscrever em um determinado serviço e receber informações [ETSI 2019]. Já para a integração do MEC com a Rede 5G será necessário o desenvolver uma API que possibilite a comunicação, redirecionamento de tráfego, registro de serviços, entre outros.

\section{Integração 5G-MEC}

O 5GS e o MEC possuem documentos técnicos que descrevem suas respectivas arquiteturas, protocolos, e todas as suas especificidades. Sendo assim, foram avaliados e usados como base Technical Reports, Group Reports, White Papers publicados pela 3GPP e ETSI que discutem as especificações das tecnologias supracitadas. Contudo, os órgãos padronizadores estão em contínua atualização destes documentos.

Para compor o ambiente de testes, foi escolhido o framework MEC EALTEdge [Akraino Wiki 2020]. Em sua arquitetura, que pode ser vista na Figura 5, é possível identificar pontos chaves da arquitetura de referência MEC, como MEP, MEC Platform Manager (MEPM) e MEO. A documentação da plataforma é rica, entretanto, foram enfrentadas dificuldades quanto a configuração dos arquivos de implantação que é toda automatizada. Neste ponto, procurou-se contato com a equipe desenvolvedora para solucionarmos os problemas. Após contato, foi possível configurá-los corretamente.

O EALTEdge disponibiliza três modelos para deployment em sua documentação: i) Completo, que utiliza três máquinas virtuais; ii) MUNO, que utiliza duas máquinas virtuais, isolando apenas o MEP, também chamado de nó de borda (Edge Node); iii) Allin-one (AIO), que utiliza apenas uma máquina virtual e concentra todos os componentes e funcionalidades nela. Dentro do contexto deste trabalho, utilizou-se o modelo AIO com o objetivo de simplificar toda a configuração e manutenção do ambiente. Este modelo utiliza uma máquina virtual com 4 CPUs, 16GB de RAM e 100GB de ROM.
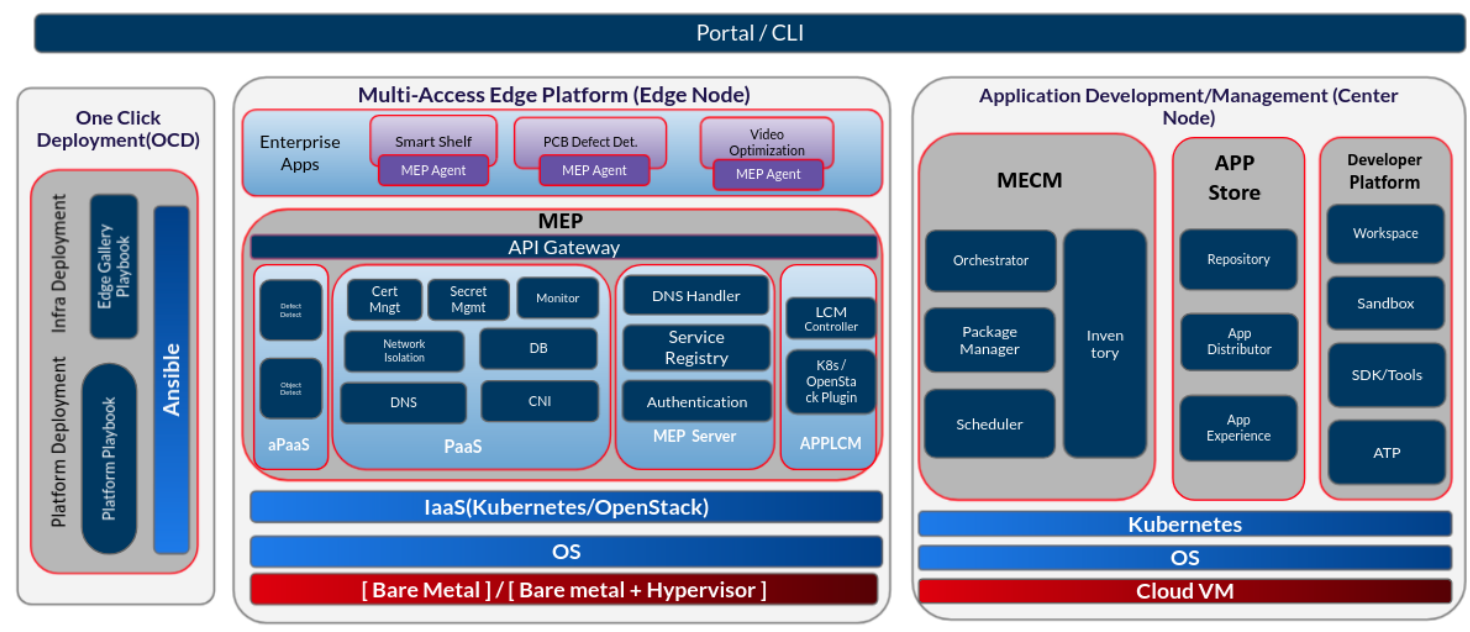

Figure 5. Arquitetura da plataforma EALTEdge. Retirada de [Akraino Wiki 2020].

A implantação de aplicações e serviços no EALTEdge são feitas usando o Edge Gallery (EG). O EG é uma plataforma, que tem como objetivo melhorar a interação entre desenvolvedor e framework, para isto utiliza um ambiente gráfico de front-end. Para 
que isto seja possível, a documentação de implantação provê um passo-a-passo para sua configuração, de forma que ao final seja possível ao usuário executar e testar o deployment de uma aplicação [Akraino Wiki 2020]. São disponibilizadas aplicações para que seja possível validar todas as configurações.

Para as redes 5G, foi utilizado o my5G-core [My5G Initiative 2020], que é um fork do free5GC [National Chiao Tung University 2021]. Assim como o EALTEdge, tem-se um deployment completo já funcional do my5G-core em ambiente virtualizado. Para isso, foi utilizada uma máquina com 2 CPUs, 8GB RAM e 160GB ROM. Com o deployment feito, passa-se à fase de configuração do core 5G, para que fosse possível permitir que a integração entre MEC e 5G Core (5GC). Na Figura 6 é apresentado o cenário de deployment e testes, com suas respectivas configurações.

A configuração do my5G-core é feita através dos arquivos de configuração de cada Network Function (NF). Sendo assim, foi alterado o endereço de cada uma delas, interligando-as por meio de uma bridge. O User Plane Function (UPF) se conecta a Session Management Function (SMF) por meio da $N 4$, que nada mais é que uma interface de rede para comunicação entre as NFs. Para a conexão do UPF com a internet foram utilizadas duas interfaces virtuais. Contudo, há uma configuração feita no SMF para que os dados sejam apontados para o servidor DNS do Google. A conexão com o MEC utiliza a mesma estratégia de duas interfaces virtuais de rede. Além das interfaces virtuais, também foi criado um namespace para que o UPF esteja isolado no ambiente, e a entrada e saída de tráfego e informações seja feita apenas utilizando as interfaces associadas a este namespace.

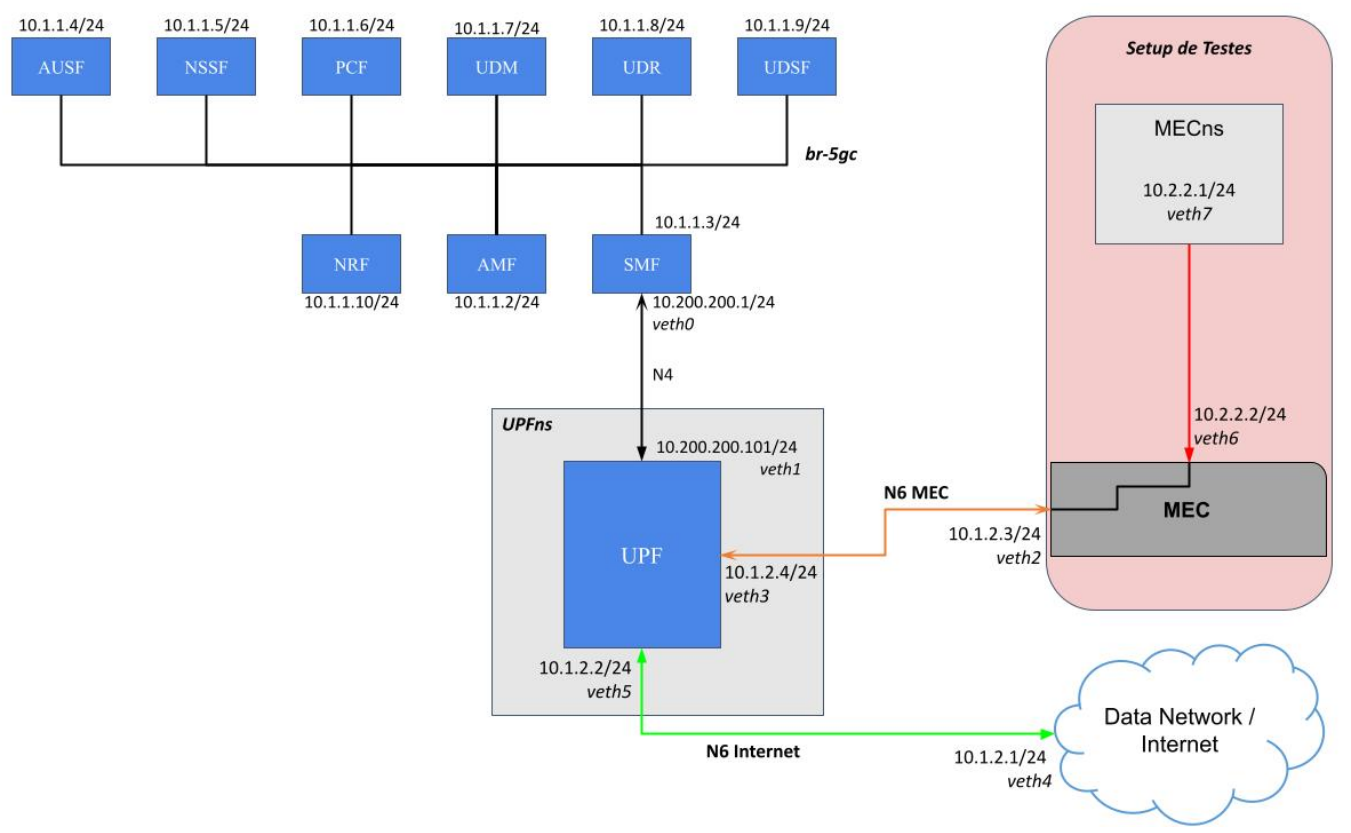

Figure 6. Setup de deployment e testes do my5G-core.

Para garantir o funcionamento de todo este ambiente, foi criado um setup de testes, destacado em vermelho na Figura 6. Para tal, criou-se um novo namespace, chamado MECns, para este ambiente esteja isolado das demais interfaces virtuais. Além disso, foi definido uma rota default entre a veth 2 e veth 6 a fim de que estas estejam conectadas. 
Após todas estas configurações, foi possível realizar um ping de dentro do MECns até a Internet e também para as NFs.

Para que estes dois frameworks se comuniquem é necessário que seja desenvolvido uma Application Program Interface (API) que forneça um serviço para comunicação entre MEC e 5GC. Decidiu-se então implementar este serviço como um componente na API Multi-Acces Traffic Sttering (MTS) descrita em [ETSI 2020]. A ETSI fornece para o desenvolvedor um OpenAPI com todas as definições de suas outras funcionalidades o qual será usado como ponto de partida. Para prover tal serviço, serão desenvolvidos dois componentes sendo eles Data Manager e Connection Manager, para receber dados e gerenciar a conexão com o 5GC. Contudo, esta API ainda está em fase de desenvolvimento.

\section{Conclusão e Próximos Passos}

Com o avanço das tecnologias e desenvolvimento de diversos serviços, é possível identificar a oportunidade para novas aplicações no cenário agrícola. Com a analise bibliográfica feita até o momento ressalta-se que a quantidade de requisitos para estas aplicações vem aumentando, tornando-se necessário meios de otimizar aplicações sensíveis ao contexto diretamente na borda da rede. Assim surge a proposta de integração de um serviço RNIS acoplado ao MEC distribuindo informações de RAN sobre o contexto dos UEs.

Os resultados alcançados até o momento serão utilizados como base para o desenvolvimento e implantação do serviço RNIS compatível com os padrões da plataforma MEC. Para isso, ainda há a necessidade de finalizar a integração entre MEC e 5GC, a partir da implementação de um serviço de comunicação entre ambos. Para tal, deve ser implementada uma API, que ao implantada no MEC, disponibilizará tal serviço. Assim que esta estiver implementada, testada e validada, serão possíveis testes em ambiente fim a fim, além de possibilitar a implantação de um serviço RNIS que também será desenvolvido.

\section{References}

3rd Generation Partnership (3GPP) (2018). Tr21.915 v15.9.0, 3rd generation partnership project. https://www.3gpp.org/ftp/Specs/archive/21_ series/21.915/. Acessado em: 06-05-2021.

Akraino Wiki (2020). Enterprise application on light weight 5g telco edge. https: //wiki.akraino.org/display/AK/Enterprise+Applicationston+ Lightweight+5G+Telco+Edge. Acessado em: 20-12-2020.

Arora, S., Frangoudis, P. A., and Ksentini, A. (2019). Exposing radio network information in a mec-in-nfv environment: the rnisaas concept. In 2019 IEEE Conference on Network Softwarization (NetSoft), pages 306-310. IEEE.

Association, G. I. P. et al. (2015). 5g vision-the 5g infrastructure public private partnership: the next generation of communication networks and services. White Paper, February.

Bonneau, V., Copigneaux, B., Probst, L., and Pedersen, B. (2017). Industry 4.0 in agriculture: Focus on iot aspects. Directorate-General Internal Market, Industry, Entrepreneurship and SMEs. 
Cepea, C. (2020). Pib do agronegócio brasileiro. https://www . cepea. esalq. usp.br/br/pib-do-agronegocio-brasileiro.aspx. Acessado em: 0605-2021.

ETSI (2019). Multi-access Edge Computing (MEC); Radio Network Information API, ETSI GS MEC 012 V2.1.1.

ETSI (2020a). Mobile technologies - 5g, 5g specs - future technology. https: / / www.etsi.org/technologies/5G. Acessado em: 08-06-2021.

ETSI (2020b). Multi-access edge computing (mec); framework and reference architecture. Technical report, $2020 \mathrm{~b}$.

ETSI, I. (2020). Multi-access edge computing (mec); traffic management apis. Technical report, 2020.

Ferreira, A. C., Ferreira, J. S., Mendes, L. L., Barbosa, T. C., and Mendes, L. L. (2019). $5 \mathrm{~g}$ iot atividade 1.1 - levantamento de aplicações do iot em áreas remotas/rurais. $C R R$ Inatel-Brasil.

Foukas, X., Patounas, G., Elmokashfi, A., and Marina, M. K. (2017). Network slicing in 5g: Survey and challenges. IEEE Communications Magazine, 55(5):94-100.

Fuentes, S., Tongson, E., and Viejo, C. G. (2021). Novel digital technologies implemented in sensory science and consumer perception. Current Opinion in Food Science.

Hu, Y. C., Patel, M., Sabella, D., Sprecher, N., and Young, V. (2015). Mobile edge computing - a key technology towards 5g. ETSI white paper, 11(11):1-16.

ITU (2021). Detailed specifications of the terrestrial radio interfaces of international mobile telecommunications-2020 (imt-2020). https://www.itu.int/rec/ R-REC-M.2150-0-202102-I/en. Recommendation ITU-R M.2150-0.

Ksentini, A. and Frangoudis, P. A. (2020). Toward slicing-enabled multi-access edge computing in 5g. IEEE Network, 34(2):99-105.

Martins, A. V. S. and Barbosa, R. A. P. (2019). O impacto da internet das coisas no agronegócio. In CLAV 2019.

Massruhá, S. M. F. S. and Leite, M. d. A. (2017). Agro 4.0-rumo à agricultura digital. In Embrapa Informática Agropecuária-Artigo em anais de congresso (ALICE). In: MAGNONI JÚNIOR, L.; STEVENS, D.; SILVA, WTL da; VALE, JMF do; PURINI, SR .

Massruhá, S. M. F. S., LEITE, M. d. A., OLIVEIRA, S. d. M., Meira, C. A. A., LUCHIARI JUNIOR, A., and BOLFE, E. (2020). Agricultura digital: pesquisa, desenvolvimento e inovação nas cadeias produtivas. Embrapa Informática AgropecuáriaLivro científico (ALICE).

My5G Initiative (2020). My5g-core. https : / / github. com/my5G/my5G-core.

National Chiao Tung University (2021). free5gc. https://www. free $5 \mathrm{gc}$. org/. Acessado em 01-06-2021.

Pham, Q.-V., Fang, F., Ha, V. N., Piran, M. J., Le, M., Le, L. B., Hwang, W.-J., and Ding, Z. (2020). A survey of multi-access edge computing in 5g and beyond: Fundamentals, technology integration, and state-of-the-art. IEEE Access, 8:116974-117017. 
Porambage, P., Okwuibe, J., Liyanage, M., Ylianttila, M., and Taleb, T. (2018). Survey on multi-access edge computing for internet of things realization. IEEE Communications Surveys \& Tutorials, 20(4):2961-2991.

Rommer, S., Hedman, P., Olsson, M., Frid, L., Sultana, S., and Mulligan, C. (2019). 5G Core Networks: Powering Digitalization. Elsevier.

Sabella, D., Vaillant, A., Kuure, P., Rauschenbach, U., and Giust, F. (2016). Mobile-edge computing architecture: The role of mec in the internet of things. IEEE Consumer Electronics Magazine, 5(4):84-91.

Silva, J. M. P. and Cavichioli, F. A. (2020). O uso da agricultura 4.0 como perspectiva do aumento da produtividade no campo. Revista Interface Tecnológica, 17(2):616-629.

Silva, J. P. L., Nery, S. W. L., Silva, R., Oliveira-Jr, A. C., Cardoso, K. V., and Both, C. B. (2021). Entendendo o núcleo $5 \mathrm{~g}$ na prática, através de uma implementação de código aberto. In SBRT 2020: Livro de Minicursos, chapter 1. Instituto Federal de Ensino, Ciência e Tecnologia da Paraíba - IFPB, Florianópolis-SC.

SILVA, J. P. L., NERY, S. W. L., SILVA, R. S., OLIVEIRA-JR, A., CARDOSO, K., and BOTH, C. B. (2020). Capítulo 1 entendendo o núcleo $5 \mathrm{~g}$ na prática, através de uma implementação de código aberto. XXXVIII Simpósio Brasileiro de Telecomunicações e Processamento de Sinais, page pp.35. SBrT2020.

Tan, Y., Han, C., Luo, M., Zhou, X., and Zhang, X. (2018). Radio network-aware edge caching for video delivery in mec-enabled cellular networks. In 2018 IEEE Wireless Communications and Networking Conference Workshops (WCNCW), pages 179-184. IEEE.

Wang, D., Chen, D., Song, B., Guizani, N., Yu, X., and Du, X. (2018). From iot to 5g i-iot: The next generation iot-based intelligent algorithms and $5 \mathrm{~g}$ technologies. IEEE Communications Magazine, 56(10):114-120. 